\title{
Deep learning detection of informative features in tau PET for Alzheimer's disease classification
}

\author{
Taeho Jo ${ }^{1,2,3}$, Kwangsik Nho ${ }^{1,2,3}$, Shannon L. Risacher ${ }^{1,2,3}$, and Andrew J. Saykin ${ }^{1,2,3^{*}}$; \\ for the Alzheimer's Neuroimaging Initiative ${ }^{a}$ \\ ${ }^{1}$ Department of Radiology and Imaging Sciences, Center for Neuroimaging, Indiana University School of \\ Medicine, Indianapolis, IN, United States \\ ${ }^{2}$ Indiana Alzheimer's Disease Research Center, Indiana University School of Medicine, Indianapolis, IN, \\ United States \\ ${ }^{3}$ Indiana University Network Science Institute, Bloomington, IN, United States \\ *Corresponding author \\ ${ }^{a}$ Data used in preparation of this article were obtained from the Alzheimer's Disease Neuroimaging \\ Initiative (ADNI) database (adni.loni.usc.edu). As such, the investigators within the ADNI contributed to \\ the design and implementation of ADNI and/or provided data but did not participate in analysis or \\ writing of this report. A complete listing of ADNI investigators can be found at: \\ http://adni.loni.usc.edu/wp-content/uploads/how_to_apply/ADNI_Acknowledgement_List.pdf
}

Email addresses:

TJ: tjo@iu.edu

KN: knho@iupui.edu

SLR: srisache@iupui.edu

AJS: asaykin@iupui.edu 


\begin{abstract}
Background:

Alzheimer's disease (AD) is the most common type of dementia, typically characterized by memory loss followed by progressive cognitive decline and functional impairment. Many clinical trials of potential therapies for $A D$ have failed, and there is currently no approved disease-modifying treatment. Biomarkers for early detection and mechanistic understanding of disease course are critical for drug development and clinical trials. Amyloid has been the focus of most biomarker research. Here, we developed a deep learning-based framework to identify informative features for AD classification using tau positron emission tomography (PET) scans.
\end{abstract}

Methods:

We analysed $\left[{ }^{18} \mathrm{~F}\right]$ flortaucipir PET image data from the Alzheimer's Disease Neuroimaging Initiative (ADNI) cohort. We first developed an image classifier to distinguish AD from cognitively normal (CN) older adults by training a 3D convolutional neural network (CNN)-based deep learning model on tau PET images ( $N=132 ; 66 \mathrm{CN}$ and $66 \mathrm{AD}$ ), then applied the classifier to images from individuals with mild cognitive impairment ( $\mathrm{MCl} ; \mathrm{N}=168$ ). In addition, we applied a layer-wise relevance propagation (LRP)based model to identify informative features and to visualize classification results. We compared these results with those from whole brain voxel-wise between-group analysis using conventional Statistical Parametric Mapping (SPM12).

Results:

The 3D CNN-based classification model of AD from CN yielded an average accuracy of $90.8 \%$ based on five-fold cross-validation. The LRP model identified the brain regions in tau PET images that contributed most to the $A D$ classification from $C N$. The top identified regions included the hippocampus, parahippocampus, thalamus, and fusiform. The LRP results were consistent with those from the voxelwise analysis in SPM12, showing significant focal $A D$ associated regional tau deposition in the bilateral temporal lobes including the entorhinal cortex. The AD probability scores calculated by the classifier were correlated with brain tau deposition in the medial temporal lobe in $\mathrm{MCl}$ participants ( $r=0.43$ for early $\mathrm{MCl}$ and $\mathrm{r}=0.49$ for late $\mathrm{MCl}$ ).

Conclusion:

A deep learning framework combining 3D CNN and LRP algorithms can be used with tau PET images to identify informative features for $A D$ classification and may have application for early detection during prodromal stages of AD. 


\section{Introduction}

The accumulation of hyperphosphorylated and pathologically misfolded tau protein is one of the cardinal and most common features in Alzheimer's disease (AD) [1-5]. The amount and spatial distribution of abnormal tau, seen pathologically as neurofibrillary tangles in brain, is closely related to the onset of cognitive decline and the progression of $A D$. The identification of morphological phenotypes of tau on in vivo neuroimaging may help to differentiate mild cognitive impairment (MCl) and $A D$ from cognitively normal older adults $(C N)$ and provide insights regarding disease mechanisms and patterns of progression [6-9].

Deep learning has been used in a variety of applications in response to the increasingly complex and growing amount of medical imaging data [10-12]. Significant efforts have been made regarding the application of deep learning to $A D$ research, but predicting $A D$ progression through deep learning using neuroimaging data has focused primarily on magnetic resonance imaging (MRI) and/or amyloid positron emission tomography (PET) $[10,13]$. However, MRI scans cannot visualize molecular pathological hallmarks of $A D$, and amyloid PET cannot, without difficulty, visualize the progression of $A D$ due to the accumulation of amyloid- $\beta$ early in the disease course with a plateau in later stages $[14,15]$.

The presence and location of pathological tau deposition in the human brain is well established $[2,3,5]$. Braak and Braak [5] analyzed AD-related neuropathology and generated a staging algorithm to describe the tau anatomical distribution $[6,8,16,17]$. Their results have been confirmed by subsequent studies showing that the topography of tau corresponds with the pathological stages of neurofibrillary tangle deposition. Cross-sectional autopsy data shows that AD-related tau pathology may begin with tau deposition in the medial temporal lobe (Braak stages I / II), then moves to the lateral temporal cortex and part of the medial parietal lobe (stage III / IV), and eventually to broader neocortical regions (V / VI).

In this study, we developed a novel deep learning-based framework that identifies the morphological phenotypes of tau deposition in tau PET images for the classification of AD from CN. Application of CNN to tau PET is novel as the spatial characteristics and interpretation are quite different compared to amyloid PET, FDG PET or MRI. In particular, the regional location and topography of tau PET signal is considered to be more important than for other molecular imaging modalities. This has implications for how CNN interacts with the complex inputs as well as for visualization of informative features. The deep learning-derived $A D$ probability scores were then applied to prodromal stages of disease including early and late mild cognitive impairment ( $\mathrm{MCl})$.

\section{Materials and Methods}

\section{Study participants}

All individuals included in the analysis were participants in the Alzheimer's Disease Neuroimaging Initiative (ADNI) cohort [18, 19]. A total of 300 ADNI participants ( $N=300 ; 66 C N, 66$ AD, 97 early mild cognitive impairment $(\mathrm{EMCl})$, and 71 late $\mathrm{MCl}(\mathrm{LMCl})$ ) with $\left[{ }^{18} \mathrm{~F}\right]$ flortaucipir PET scans were available for analysis [1]. Genotyping data were also available for all participants [19]. Informed consent was obtained for all subjects, and the study was approved by the relevant institutional review board at each data acquisition site. 


\section{Alzheimer's Disease Neuroimaging Initiative (ADNI)}

$A D N I$ is a multi-site longitudinal study investigating early detection of $A D$ and tracking disease progression using biomarkers (MRI, PET, other biological markers, and clinical and neuropsychological assessment) [1]. Demographic information, PET and MRI scan data, and clinical information are publicly available from the ADNI data repository (http://www.loni.usc.edu/ADNI/).

\section{Imaging processing}

Pre-processed $\left[{ }^{18} \mathrm{~F}\right]$ flortaucipir PET scans $(\mathrm{N}=300)$ were downloaded from the ADNI data repository, one scan per individual. Scans were normalized to Montreal Neurologic Institute (MNI) space using parameters generated from segmentation of the T1-weighted MRI scan in Statistical Parametric Mapping v12 (SPM12) (www.fil.ion.ucl.ac.uk/spm/). Standard uptake value ratio (SUVR) images were then created by intensity-normalization using a cerebellar crus reference region.

\section{Deep learning method for AD classification}

Deep learning is a subset of machine learning that has been applied in various fields [20, 21]. Deep learning uses a back-propagation procedure [22], which utilizes gradient descent for the efficient error functions and gradient computing [10, 23-26]. The weights are updated after the initial error value is calculated by the least squares method until the differential value becomes 0 , as in the following formula:

$$
W_{i j}(t+1)=W_{i j} t-\frac{\partial \operatorname{Error}_{o u t}}{\partial W_{i j}}
$$

Here, $W_{i j} t$ is a current weight of neuron $j$ in layer $i$, and $W_{i j}(t+1)$ is the next. Errory $Y_{\text {out }}$ is the sum of errors that are known through the given data. $W_{i j}$ can be calculated by the chain rule as follows:

$$
\frac{\partial \operatorname{Error}_{\text {out }}}{\partial W_{i j}}=\frac{\partial \operatorname{Error}_{\text {out }}}{\partial y_{o j}} \cdot \frac{\partial y_{o j}}{\partial n e t_{i}} \cdot \frac{\partial n e t_{i}}{\partial w_{i j}}
$$

Net is a sum of weights and bias, and $Y_{o j}$ is an output of neuron $j$. Convolutional Neural Network (CNN) is a method of inserting convolution and pooling layers to the basic structure of this neural network to reduce complexity. Since CNN is widely used in the field of visual recognition, we used a CNN method for the classification of AD from CN [27]. The overall architecture of 3D CNN that we used is shown in Fig. 1. To avoid excessive epochs that can lead to overfitting, an early stopping method was applied to cease training if the model did not show improvement over 10 iterations. The learning rate of 0.0001 and Adam, a first-order gradient based probabilistic optimization algorithm [28] with a batch size of 4, were used for training a model. Feature maps $(8,16$, and 32 features) were extracted from three hidden layers, with Maxpool3D and BatchNorm3D applied to each layer [29]. Dropout (0.4) was applied to the second and third layers. The training model was applied to the validation set, and each image was determined as AD or CN. Five-fold cross validation was applied, where $60 \%$ of the entire data set was used for training, $20 \%$ for testing, and $20 \%$ for validating. Training images were augmented by three criteria: flipping the image data, shifting the position within two voxels, and shifting the position simultaneously with the flip. Each fold was repeated four times for a robustness check, and the mean 
accuracy of the four repeats was used as the final accuracy. Pytorch 1.0.1 was used to design neural networks and load pre-trained weights, and all of the programs were run on Python 3.5.

\section{Application of the AD-CN derived classification model to $\mathrm{MCl}$}

After an $A D$ classification model was constructed using $A D$ and $C N$ groups, the model was applied to the tau PET scans from the $\mathrm{MCl}$ participants to calculate $A D$ probability scores. The AD probability scores were distributed from 0 to 1 , and individuals with $A D$ probability scores closer to 1 were classified as having $A D$ characteristics, and individuals with scores closer to 0 were classified as having $C N$ characteristics.

\section{Identification of informative features for AD classification}

We applied a layer-wise relevance propagation (LRP) algorithm to identify informative features and visualize the classification results [30,31]. The LRP algorithm is used to determine the contribution of a single pixel of an input image to the specific prediction in the image classification task (for full details of the LRP algorithm, see [30]).

The output $x_{j}$ of a neuron $j$ is calculated by a nonlinear activation function $g$ and function $h$ such as

$$
x_{j}=g_{j}\left(\sum_{i} h_{i j}\left(x_{i}\right)\right)
$$

If the relevance score $\mathrm{R}$ of the $j$ neuron in the layer $I+1$ sets to $R_{i \leftarrow j}^{(l, l+1)}$, the relevance score $\mathrm{R}$ sent to the neuron $i$ in the layer $I$ will be represented as $R_{i \leftarrow j}^{(l, l+1)}$. So, the input value of the neuron $j$ can be expressed as the following equation:

$$
R_{i \leftarrow j}^{(l, l+1)}=\sum_{i \in(l)} R_{i \leftarrow j}^{(l, l+1)}
$$

Bach, et al. [30] proposed the following formula for calculating $R_{i \leftarrow j}^{(l, l+1)}$ :

$$
R_{i \leftarrow j}^{(l, l+1)}=\left((1+\beta) \frac{z_{i j}^{+}}{z_{j}^{+}}-\beta \frac{z_{i j}^{-}}{z_{j}^{-}}\right) R_{j}^{(l+1)}
$$

Here, $z_{i j}^{+}$represents the positive input that the node $i$ contributes to the node $j$, and $z_{i j}^{-}$represents the negative input. The variable $\beta$ that ranges from 0 to 1 controls the inhibition of the relevance redistribution. A larger $\beta$ value (e.g. $\beta=1$ ) makes the heat map clearer [31]. In this experiment, we set $\beta=1$.

\section{Whole-brain imaging analysis}

A voxel-wise whole brain analysis to identify brain regions in the tau PET SUVR images showing significantly higher tau deposition in $A D$ relative to $C N$ was conducted in SPM12. The analysis was masked for grey plus white matter. The voxel-wise family-wise error (FWE) correction was applied at $p<$ 0.05 , with a cluster size of $\geq 50$ voxels for adjustment for multiple comparisons.

\section{Results}

In the analysis, $300 \mathrm{ADNI}$ participants (66 CN, $66 \mathrm{AD}, 97 \mathrm{EMCl}$, and $71 \mathrm{LMCl}$ ) who had baseline tau PET scans were used. Sample demographics were given in Table 1. 


\section{Classification of $A D$ from $C N$}

We developed an image classifier to distinguish $A D$ from $C N$ by training a $3 D$ CNN-based deep learning model on tau PET images. As the number of individuals with $A D$ who had tau PET data were smaller than those of $\mathrm{CN}$, we chose the same number of $\mathrm{CN}$ randomly (66 CN) in order to train a classifier with a balanced dataset. In the binary classification problem, it is a well-known issue that detecting disease when the majority of the applicants are healthy, the majority group may be referred as cases, causing biased classification[32]. So we used a random under-sampling (RUS) method to decrease samples from the majority group. All analyses were performed using five-fold cross-validation to reduce the likelihood of overfitting. Ultimately, cross validation in a novel independent data set will be important when such data becomes available. The classification accuracy is shown in Table 2. Our deep learning-based classification model of AD from CN yielded an average accuracy of $90.8 \%$ and a standard deviation of $2 \%$ from five-fold cross-validation (Table 2).

\section{Identification of informative features for $A D$ classification}

The LRP algorithm generated relevance heatmaps in the tau PET image to identify which brain regions play a significant role in a deep learning-based $A D$ classification model. After selecting an $A D$ classification model with the highest accuracy in each fold, we generated five heatmaps and selected the top ten regions with the highest contribution. Figure $2 \mathrm{~A}$ shows a visualization of the relevance heatmap in three orientations of our 3D CNN-based classification of AD from CN. The heatmap displays the primary brain regions that contributed to the classification, color-coded with increasing values from red to yellow. The colored regions in the heatmap include the hippocampus, parahippocampal gyrus, thalamus, and fusiform gyrus (Fig. 2A). For comparison with our 3D CNN-based LRP results, Figure 2B shows the results of whole brain voxel-wise analysis in SPM12 to identify brain regions where there are significant differences between $A D$ and $C N$ in brain tau deposition (FWE corrected $p$-value $<0.05$; minimum cluster size $(k)=50)$. AD had significantly higher tau deposition in widespread regions including the bilateral temporal lobes with global maximum differences in the right and left parahippocampal regions, compared to $C N$ (Fig. 2B). The informative regions for AD classification in the LRP results is very similar to those found using SPM12, but the 3D CNN-based LRP identified smaller focal regions.

\section{Classification of $\mathrm{MCl}$ based on the AD-CN classification model}

We calculated the $A D$ probability scores of $\mathrm{MCl}$ participants ( $97 \mathrm{EMCl}$ and $71 \mathrm{LMCl}$, separately) using the classification model generated above. Figure $\mathbf{3 A}$ and Figure $\mathbf{3 B}$ show scatter plots between the $A D$ probability scores of $\mathrm{EMCl}$ and $\mathrm{LMCl}$, respectively, with bilateral mean tau deposition in the medial temporal lobe (includes the entorhinal cortex, fusiform, and parahippocampal gyri). The correlation coefficients were $\mathrm{R}=0.43$ for $\mathrm{EMCl}$ and $\mathrm{R}=0.49$ for $\mathrm{LMCl}$, with greater tau deposition levels in the medial temporal lobe associated with higher $A D$ probability scores. Figure $3 C$ and Figure $3 D$ show mean tau accumulation in the medial temporal cortex of $\mathrm{EMCl}$ and $\mathrm{LMCl}$, respectively, for participants with $\mathrm{AD}$ probability score ranges $(0 \leq A D$ probability score $\leq 0.05$ versus $0.95 \leq A D$ probability score $\leq 1.00$, the ranges to which $65 \%$ of $\mathrm{EMCl}$ and $62 \%$ of $\mathrm{LMCl}$ belong ; $0 \leq \mathrm{AD}$ probability score $<0.5$ versus $0.5<\mathrm{AD}$ probability score $\leq 1.00$ ). In $\mathrm{EMCl}$ (Fig. $3 \mathrm{C}$ ), a comparison between participants with $0 \leq A D$ probability score $\leq 0.05$ and those with $0.95 \leq A D$ probability score $\leq 1.00$ yielded a difference of 0.19 SUVR in the 
medial temporal lobe. In LMCI (Fig. 3D), the comparison of participants with low AD probability scores (0 $\leq A D$ probability score $\leq 0.05)$ and $L M C l$ with high $A D$ probability scores $(0.95 \leq A D$ probability score $\leq$ 1.00 ) yielded a difference of 0.26 SUVR in the medial temporal cortex. Whole brain voxel-wise analysis in SPM12 was performed to identify brain regions showing difference between tau deposition between $\mathrm{MCl}$ participants with low $A D$ probability scores $(0 \leq A D$ probability score $\leq 0.05)$ and those with high $A D$ probability scores (0.95 $\leq A D$ probability score $\leq 1.00$ ). In EMCI (Fig. 4A, C) and LMCI (Fig. 4B, D), voxelwise analysis identified significant group differences in the bilateral temporal lobes including the entorhinal cortex. In addition, the differences in tau deposition were more widespread in LMCI compared to $\mathrm{EMCl}$ (Fig. 4).

\section{Discussion}

We developed a deep learning framework for detecting informative features in tau PET for the classification of Alzheimer's disease. After training a 3D CNN-based AD/CN classifier on 132 $\left[{ }^{18} \mathrm{~F}\right]$ flortaucipir PET images to distinguish $A D$ with $>90 \%$ accuracy, heatmaps were generated by a LRP algorithm to show the most important regions for the classification. This model was then applied to $\left[{ }^{18} \mathrm{~F}\right]$ flortaucipir PET images from $168 \mathrm{MCl}$ to classify them into "AD similar" and "CN similar" groups for further investigation of the morphological characteristics of the tau deposition.

Maass, et al. [7] examined the key regions of in vivo tau pathology in ADNI using a data-driven approach and determined that the major regions contributing to a high global tau signal mainly overlapped with Braak stage III ROIs (i.e., amygdala, parahippocampal gyri and fusiform). Our deep learning-based results correspond well to the pattern reported by Maass, et al. [7] on a more limited data set.

It is noteworthy that stages III / IV can be seen in both CN and AD patients, while stages I / II are common in $\mathrm{CN}$ and stages $\mathrm{V} / \mathrm{VI}$ are common for $\mathrm{AD}$ patients [3]. Thus, it is difficult to predict $A D$ by measuring tau deposition in stage III / IV ROIs, highlighting the importance of understanding the morphological characteristics of tau. Our heatmaps, which visualized the regions driving the classification of $A D$ and $C N$ using deep learning on tau PET images, showed a distribution pattern similar group differences in tau deposition between $A D$ and $C N$ assessed using voxel-wise analysis in SPM12. This finding indicates that the deep learning classifier used the morphological characteristics of the tau distribution for classifying $A D$ from $C N$. In particular, the heatmaps show that the hippocampus, parahippocampal gyrus, thalamus and fusiform gyrus were primarily used to classify AD from CN. These results support existing research showing that tau accumulation in memory-related areas play an important role in the development of $A D[33,34]$.

Early, accurate and efficient diagnosis of $A D$ is important for initiation of effective treatment. Prognostic prediction of the likelihood of conversion of $\mathrm{MCl}$ to $A D$ plays a significant role in therapeutic development and ultimately will be important for effective patient care. Thus, the CN vs. AD classifier was used to generate a score of whether the tau distribution in $\mathrm{MCl}$ participants was similar or different from that seen in $A D$. When the $A D$ probability score generated by the classifier was high suggesting high similarity to $A D$, the $\mathrm{MCl}$ participants generally had the characteristic tau morphology seen in $A D$. In addition, we assessed applied this method to both $\mathrm{EMCl}$ and $\mathrm{LMCl}$ participants. Pearson correlation 
coefficients between AD probability scores and bilateral mean of SUVR in the medial temporal lobe were $\mathrm{R}=0.43$ for $\mathrm{EMCl}$ and $\mathrm{R}=0.49$ for $\mathrm{LMCl}$. These findings indicate that the tau deposition difference between the lower $5 \%$ and upper $95 \%$ of $\mathrm{LMCl}$ participants was $7.1 \%$ more than the difference between the lower $5 \%$ and upper $95 \%$ of $\mathrm{EMCl}$ participants. Thus, the classifier determined that the tau deposition of $\mathrm{LMCl}$ participants is more similar to those seen in $A D$ than that of $\mathrm{EMCl}$ participants. The is in line with numerous reports of biomarkers in late $\mathrm{MCl}$ where there is considerable overlap with early stage AD pathology.[18]

\section{Conclusion}

Deep learning can be used to classify tau PET images from AD patients versus controls. Furthermore, this classifier can score the tau distribution by its similarity to $A D$ when applied to scans from older individuals with $\mathrm{MCl}$. A deep learning derived $\mathrm{AD}$-like tau deposition pattern may be useful for early detection of disease during the prodromal or possibly even preclinical stages of $A D$ on an individual basis. Advances in predictive modelling are needed to develop accurate precision medicine tools for $A D$ and related neurodegenerative disorders, and further developments can be expected with inclusion of multimodality data sets and larger samples. 


\section{Declarations}

- Ethics approval and consent to participate

Ethics approval is not required as the human data were publicly available by ADNI website, and all the data are not identifiable.

\section{- Consent for publication}

Not applicable.

\section{- Availability of data and materials}

The datasets used and analyzed during the study are available in the ADNI LONI repository, http://adni.loni.usc.edu/

\section{- Competing interests}

The authors declare that they have no competing interests.

\section{- Funding}

This work was supported, in part, by grants from the National Institutes of Health (NIH) and include the following sources: P30 AG010133, R01 AG019771, R01 AG057739, R01 CA129769, R01 LM012535, R03 AG054936, K01 AG049050, R01 AG061788.

\section{- Authors' contributions}

TJ, KN, and AS: conceptualization and study design. TJ, SR: data collection. TJ: conducted the experiment and analysis. TJ, KN: drafting manuscript. TJ, KN, SR, and AS: revision of the manuscript for important scientific content and final approval.

\section{- Acknowledgements}

Data collection and sharing for this project was funded by the Alzheimer's Disease Neuroimaging Initiative (ADNI) (National Institutes of Health Grant U01 AG024904) and DOD ADNI (Department of Defense award number W81XWH-12-2-0012). ADNI is funded by the National Institute on Aging, the National Institute of Biomedical Imaging and Bioengineering, and through generous contributions from the following: AbbVie, Alzheimer's Association; Alzheimer's Drug Discovery Foundation; Araclon Biotech; BioClinica, Inc.; Biogen; Bristol-Myers Squibb Company; CereSpir, Inc.; Cogstate; Eisai Inc.; Elan Pharmaceuticals, Inc.; Eli Lilly and Company; Eurolmmun; F. Hoffmann-La Roche Ltd and its affiliated company Genentech, Inc.; Fujirebio; GE Healthcare; IXICO Ltd.; Janssen Alzheimer Immunotherapy Research \& Development, LLC.; Johnson \& Johnson Pharmaceutical Research \& Development LLC.; Lumosity; Lundbeck; Merck \& Co., Inc.; Meso Scale Diagnostics, LLC.; NeuroRx Research; Neurotrack Technologies; Novartis Pharmaceuticals Corporation; Pfizer Inc.; Piramal Imaging; Servier; Takeda Pharmaceutical Company; and Transition Therapeutics. The Canadian Institutes of Health Research is providing funds to support ADNI clinical sites in Canada. Private 
sector contributions are facilitated by the Foundation for the National Institutes of Health (www.fnih.org). The grantee organization is the Northern California Institute for Research and Education, and the study is coordinated by the Alzheimer's Therapeutic Research Institute at the University of Southern California. ADNI data are disseminated by the Laboratory for Neuro Imaging at the University of Southern California.

The authors are grateful to Paula Bice, PhD for editorial assistance. 


\section{Figures and Tables}

\begin{tabular}{l|llll|l}
\hline & AD & CN & EMCI & LMCl & Total \\
\hline \hline $\mathbf{n}$ & 66 & 66 & 97 & 71 & 300 \\
Age & 76.6 & 69.3 & 73.4 & 73.4 & 73.2 \\
(SD) & $(8.9)$ & $(5.4)$ & $(7.5)$ & $(8.0)$ & $(7.5)$ \\
\% male & $56.1 \%$ & $40.0 \%$ & $37.9 \%$ & $66.7 \%$ & $50.2 \%$ \\
\hline Education & 15.8 & 17.2 & 16.3 & 16.4 & 16.4 \\
(SD) & $(2.5)$ & $(2.1)$ & $(2.8)$ & $(2.5)$ & $(2.5)$ \\
\% amyloid+ & $90.9 \%$ & $27.3 \%$ & $40.9 \%$ & $48.5 \%$ & $51.9 \%$ \\
\% ApoE4 carriers & $51.5 \%$ & $25.7 \%$ & $35.1 \%$ & $28.2 \%$ & $35.1 \%$ \\
\hline
\end{tabular}

Table 1. Demographic information 


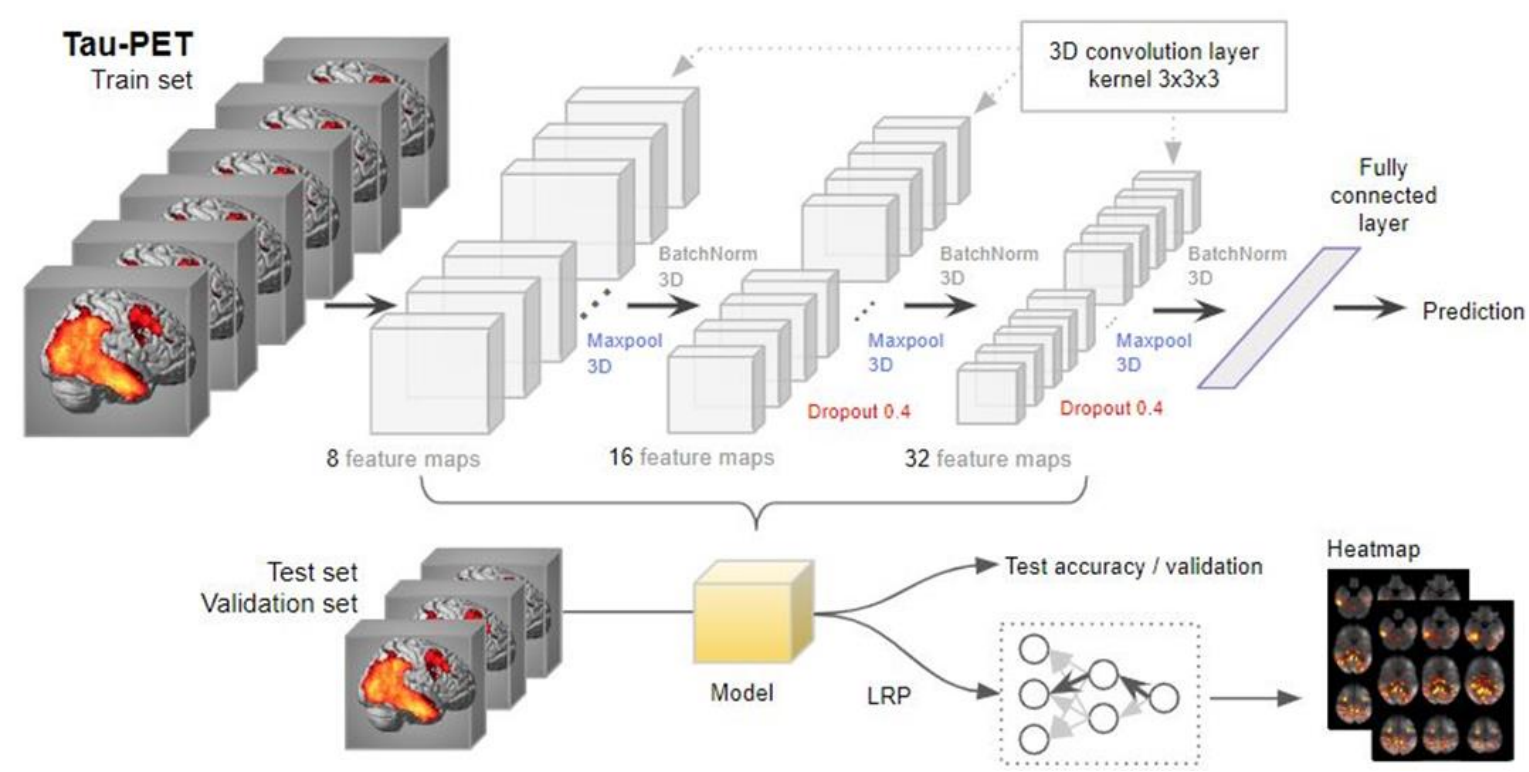

Fig. 1. 3D convolutional neural network (3D-CNN)-based and layer-wise relevance propagation (LRP)based framework for the classification of Alzheimer's disease and the identification of informative features. 


\begin{tabular}{l||lll|lll|ll|ll|l||l|l|l}
\hline & Train & Test & Val & Acc. r1 & epoch & Acc. r2 & epoch & Acc. r3 & epoch & Acc. r4 & epoch & Mean acc. & SD \\
\hline fold1 & $78(312)$ & 28 & 26 & 85.7 & 23 & 92.9 & 24 & 89.3 & 21 & 85.7 & 27 & 88.4 & 3.0 \\
fold2 & $78(312)$ & 28 & 26 & 96.2 & 20 & 96.2 & 21 & 92.3 & 21 & 88.5 & 22 & 93.3 & 3.2 \\
fold3 & $80(320)$ & 26 & 26 & 100 & 35 & 92.3 & 28 & 88.5 & 27 & 88.5 & 30 & 92.3 & 4.7 \\
fold4 & $80(320)$ & 26 & 26 & 92.3 & 24 & 88.5 & 31 & 88.5 & 38 & 88.5 & 29 & 89.4 & 1.7 \\
fold5 & $80(320)$ & 26 & 26 & 92.3 & 50 & 80.8 & 36 & 96.2 & 35 & 92.3 & 34 & 90.4 & 5.8 \\
\hline
\end{tabular}

Table 2. Results of 5 -fold cross validation. The numbers in parentheses are the training images after applying augmentation. In addition to the test set, a separate set of $20 \%$ independent validations were generated for each fold to ensure the robustness of the experiments. The experiment was repeated four times for each fold (Acc. $r 1 \sim r 4$ ), and the mean accuracy was considered as the final accuracy of the folds. If the accuracy improvement for the test set did not occur up to 10 times, the training was stopped (epoch). 


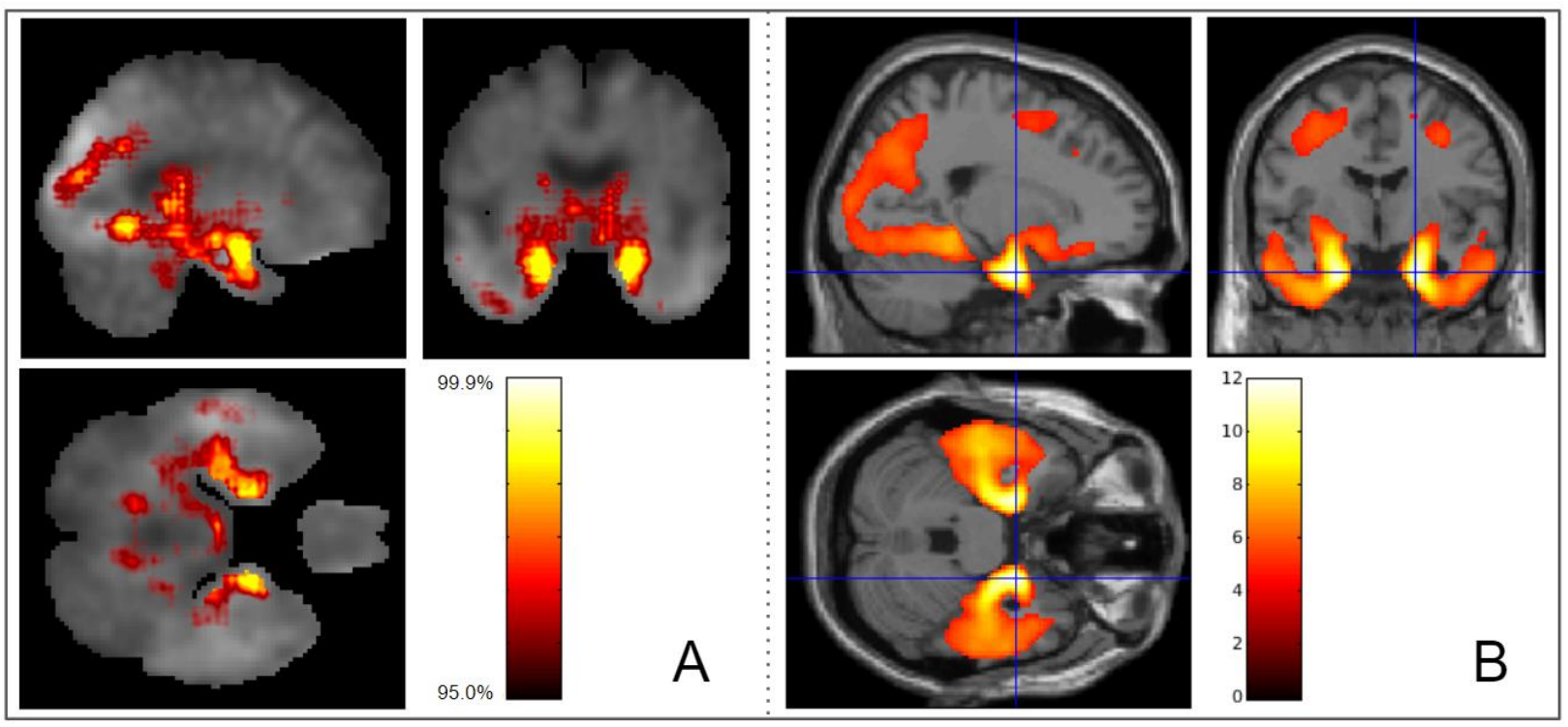

Fig 2. Heatmaps of $3 D-C N N$ classifications compared to voxel-wise group difference maps between $A D$ and CN participant groups. A. Relevance heatmaps of 3D-CNN classification of AD and CN. The bright areas represent the regions that most contribute to the CN/AD classification in CNN. Selected regions with the highest contribution include the hippocampus, parahippocampal gyrus, thalamus, fusiform gyrus, and diencephalon. B. SPM maps show similar regions of the brain as the 3D-CNN maps where tau deposition is significantly higher in the AD group compared to the CN group (Voxel-wise FWE-corrected $p$-value $<0.05$; minimum cluster size $(k)=50)$. 

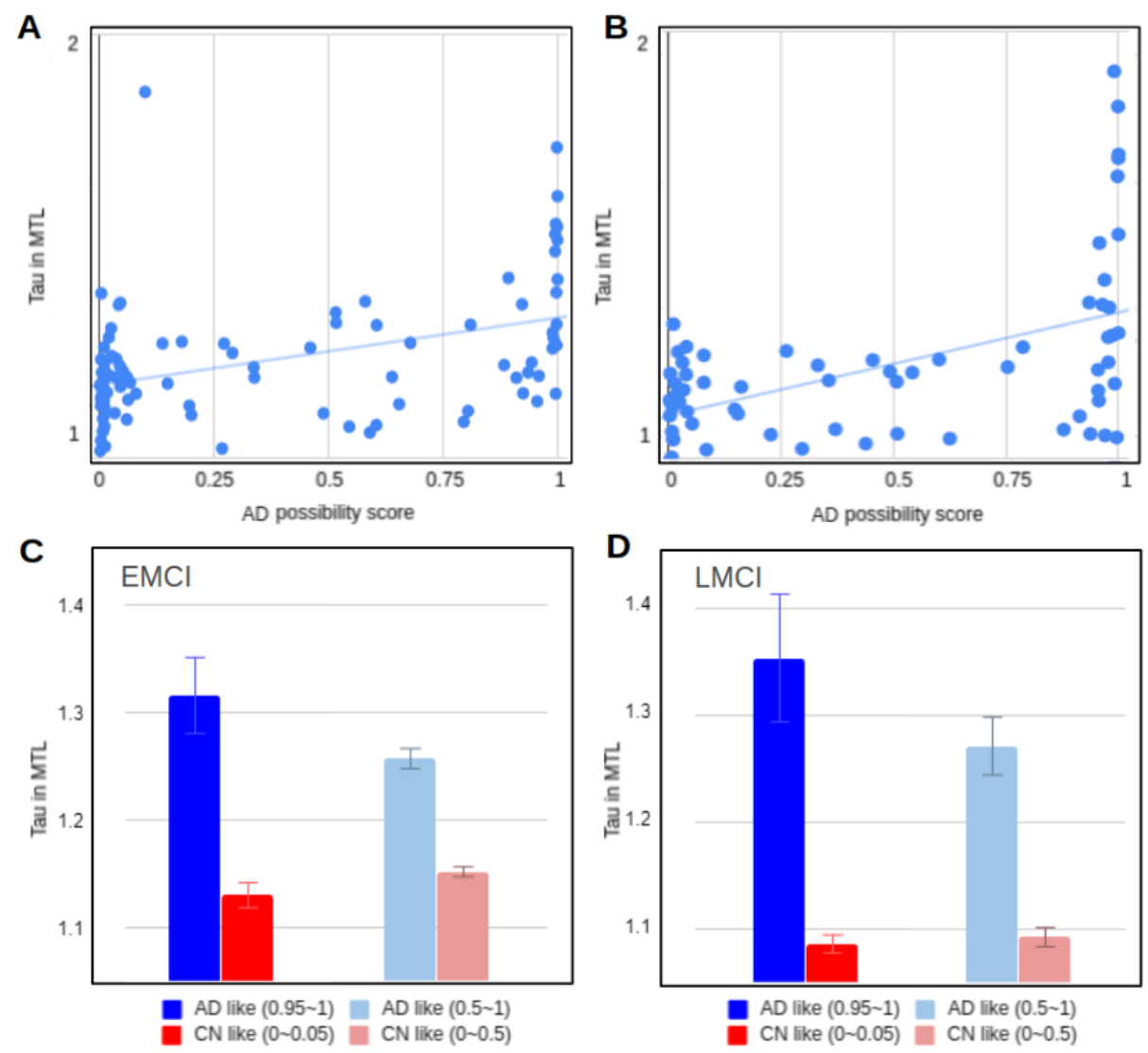

Fig. 3. Results of scoring all the images through the classifier and comparing the scores to the tau accumulation in the MTL region. Correlation of DL score with the amount of tau accumulated in the MTL region was $\mathrm{R}=0.43$ for $\mathrm{EMCl}(\mathrm{A})$ and $\mathrm{R}=0.49$ for $\mathrm{LMCl}(\mathrm{B})$. The red and blue bar chart in $\mathrm{C}$ and $\mathrm{D}$ show the average of the tau amounts of the image with the DL score of $5 \%$ and the image of the top $95 \%$. The charts in light red and light blue in C and D are the result of averaging the bottom $50 \%$ and top $50 \%$ of the images. 

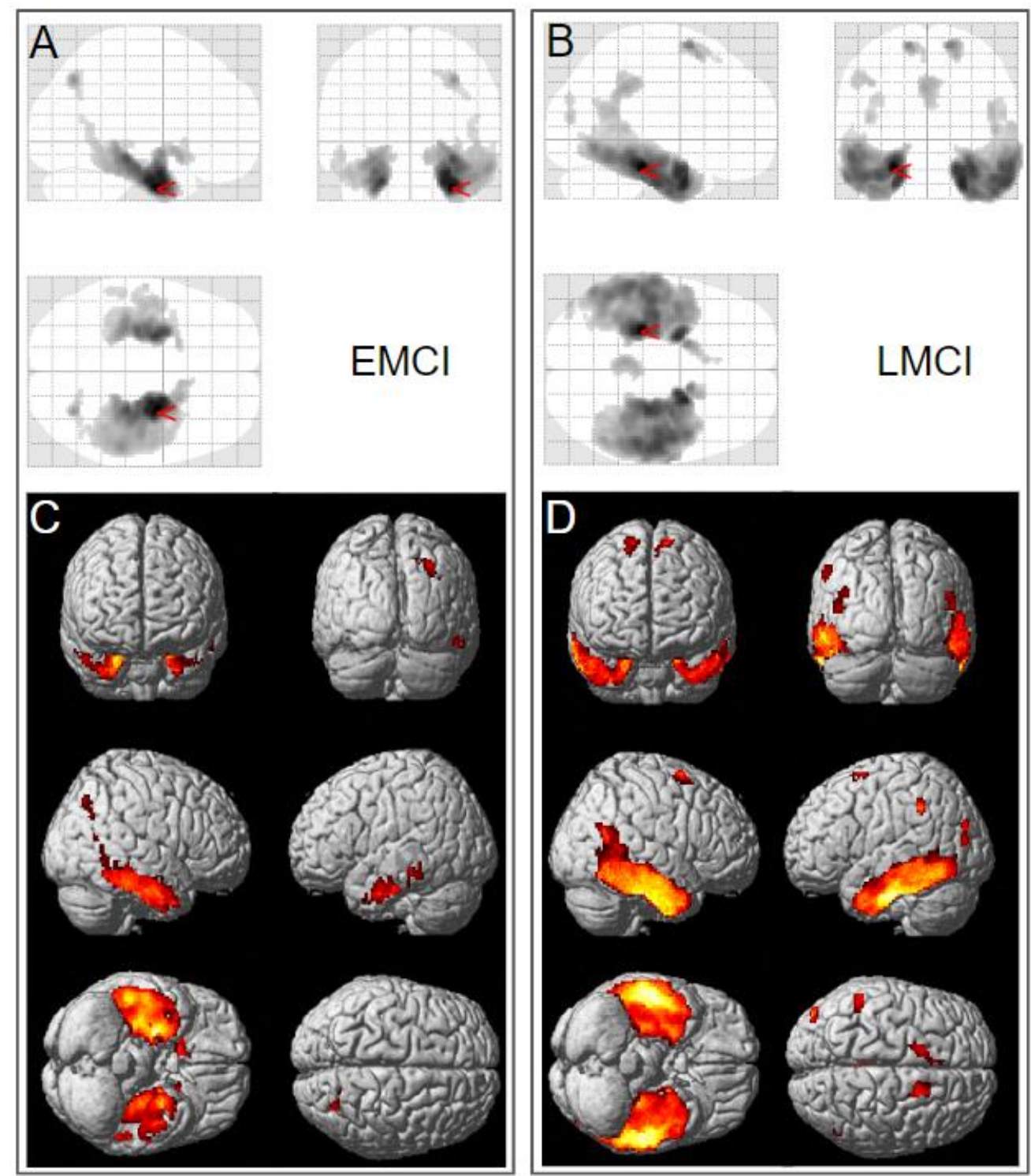

Fig. 4. Voxel-wise difference between $\mathrm{MCl}$ participants with $\mathrm{AD}$-like tau patterns and $\mathrm{CN}$-like tau patterns defined using the 3D-CNN classifier. Significantly greater tau was observed in $\mathrm{EMCl}(\mathrm{A}, \mathrm{C})$ and $\operatorname{LMCl}(B, D)$ with high $A D$ probability ("AD-like," $0.95 \leq A D$ probability score $\leq 1.00$ ) relative to the low $A D$ probability group ("CN-like," $0 \leq A D$ probability score $\leq 0.05$ ). Voxel-wise significance maps are displayed at FWE corrected $p$-value $<0.05$; minimum cluster size $(k)=50$. 


\section{Reference}

1. Weiner MW, Veitch DP, Aisen PS, Beckett LA, Cairns NJ, Green RC, Harvey D, Jack Jr CR, Jagust W, Morris JC: The Alzheimer's Disease Neuroimaging Initiative 3: Continued innovation for clinical trial improvement. Alzheimer's \& Dementia 2017, 13(5):561-571.

2. Cho H, Choi JY, Hwang MS, Kim YJ, Lee HM, Lee HS, Lee JH, Ryu YH, Lee MS, Lyoo CH: In vivo cortical spreading pattern of tau and amyloid in the Alzheimer disease spectrum. Annals of neurology 2016, 80(2):247-258.

3. Schöll M, Lockhart SN, Schonhaut DR, O'Neil JP, Janabi M, Ossenkoppele R, Baker SL, Vogel JW, Faria J, Schwimmer HD: PET imaging of tau deposition in the aging human brain. Neuron 2016, 89(5):971-982.

4. Risacher SL, Fandos N, Romero J, Sherriff I, Pesini P, Saykin AJ, Apostolova LG: Plasma amyloid beta levels are associated with cerebral amyloid and tau deposition. Alzheimer's \& Dementia: Diagnosis, Assessment \& Disease Monitoring 2019, 11:510-519.

5. Braak H, Braak E: Neuropathological stageing of Alzheimer-related changes. Acta neuropathologica 1991, 82(4):239-259.

6. Johnson KA, Schultz A, Betensky RA, Becker JA, Sepulcre J, Rentz D, Mormino E, Chhatwal J, Amariglio $\mathrm{R}$, Papp K: Tau positron emission tomographic imaging in aging and early $A$ Izheimer disease. Annals of neurology 2016, 79(1):110-119.

7. Maass A, Landau S, Baker SL, Horng A, Lockhart SN, La Joie R, Rabinovici GD, Jagust WJ, Initiative AsDN: Comparison of multiple tau-PET measures as biomarkers in aging and Alzheimer's disease. Neuroimage 2017, 157:448-463.

8. Villemagne VL, Fodero-Tavoletti MT, Masters $\mathrm{CL}$, Rowe CC: Tau imaging: early progress and future directions. The Lancet Neurology 2015, 14(1):114-124.

9. Deters KD, Risacher SL, Kim S, Nho K, West JD, Blennow K, Zetterberg H, Shaw LM, Trojanowski $\mathrm{JQ}$, Weiner MW: Plasma tau association with brain atrophy in mild cognitive impairment and Alzheimer's disease. Journal of Alzheimer's Disease 2017, 58(4):1245-1254.

10. Jo T, Nho K, Saykin AJ: Deep learning in Alzheimer's disease: diagnostic classification and prognostic prediction using neuroimaging data. Frontiers in aging neuroscience 2019, 11:220.

11. Litjens G, Kooi T, Bejnordi BE, Setio AAA, Ciompi F, Ghafoorian M, Van Der Laak JA, Van Ginneken $\mathrm{B}$, Sánchez $\mathrm{Cl}$ : A survey on deep learning in medical image analysis. Medical image analysis 2017, 42:60-88.

12. Shen D, Wu G, Suk H-I: Deep learning in medical image analysis. Annual review of biomedical engineering 2017, 19:221-248.

13. Islam J, Zhang Y: Understanding 3D CNN Behavior for Alzheimer's Disease Diagnosis from Brain PET Scan. arXiv preprint arXiv:191204563 2019.

14. Palmqvist S, Schöll M, Strandberg O, Mattsson N, Stomrud E, Zetterberg H, Blennow K, Landau S, Jagust $W$, Hansson $O$ : Earliest accumulation of $\beta$-amyloid occurs within the default-mode network and concurrently affects brain connectivity. Nature communications 2017, 8(1):1-13.

15. Panza F, Lozupone M, Logroscino G, Imbimbo BP: A critical appraisal of amyloid- $\boldsymbol{\beta}$-targeting therapies for Alzheimer disease. Nature Reviews Neurology 2019, 15(2):73-88.

16. Braak H, Alafuzoff I, Arzberger T, Kretzschmar H, Del Tredici K: Staging of Alzheimer diseaseassociated neurofibrillary pathology using paraffin sections and immunocytochemistry. Acta neuropathologica 2006, 112(4):389-404.

17. Schwarz AJ, Yu P, Miller BB, Shcherbinin S, Dickson J, Navitsky M, Joshi AD, Devous Sr MD, Mintun MS: Regional profiles of the candidate tau PET ligand 18 F-AV-1451 recapitulate key features of Braak histopathological stages. Brain 2016, 139(5):1539-1550. 
18. Veitch DP, Weiner MW, Aisen PS, Beckett LA, Cairns NJ, Green RC, Harvey D, Jack Jr CR, Jagust W, Morris JC: Understanding disease progression and improving Alzheimer's disease clinical trials: Recent highlights from the Alzheimer's Disease Neuroimaging Initiative. Alzheimer's \& Dementia 2019, 15(1):106-152.

19. Saykin AJ, Shen L, Yao X, Kim S, Nho K, Risacher SL, Ramanan VK, Foroud TM, Faber KM, Sarwar $\mathrm{N}$ : Genetic studies of quantitative $\mathrm{MCl}$ and $\mathrm{AD}$ phenotypes in ADNI: Progress, opportunities, and plans. Alzheimer's \& Dementia 2015, 11(7):792-814.

20. LeCun Y, Bengio $Y$, Hinton G: Deep learning. nature 2015, 521(7553):436-444.

21. Richiardi J, Altmann A, Milazzo A-C, Chang C, Chakravarty MM, Banaschewski T, Barker GJ, Bokde AL, Bromberg $\mathrm{U}$, Büchel C: Correlated gene expression supports synchronous activity in brain networks. Science 2015, 348(6240):1241-1244.

22. Hecht-Nielsen R: Theory of the backpropagation neural network. In: Neural networks for perception. Elsevier; 1992: 65-93.

23. Rumelhart DE, Hinton GE, Williams RJ: Learning representations by back-propagating errors. nature 1986, 323(6088):533-536.

24. Bishop CM: Neural networks for pattern recognition: Oxford university press; 1995.

25. Ripley BD: Pattern recognition and neural networks: Cambridge university press; 2007.

26. Schalkoff RJ: Artificial neural networks: McGraw-Hill Higher Education; 1997.

27. Krizhevsky A, Sutskever I, Hinton GE: Imagenet classification with deep convolutional neural networks. In: Advances in neural information processing systems: 2012. 1097-1105.

28. Kingma DP, Ba J: Adam: A method for stochastic optimization. arXiv preprint arXiv:14126980 2014.

29. loffe S, Szegedy C: Batch Normalization: Accelerating Deep Network Training by Reducing Internal Covariate Shift. In: International Conference on Machine Learning: 2015. 448-456.

30. Bach S, Binder A, Montavon G, Klauschen F, Müller K-R, Samek W: On pixel-wise explanations for non-linear classifier decisions by layer-wise relevance propagation. PloS one 2015, 10(7).

31. Böhle $M$, Eitel F, Weygandt $M$, Ritter K: Layer-wise relevance propagation for explaining deep neural network decisions in mri-based alzheimer's disease classification. Frontiers in aging neuroscience 2019, 11:194.

32. Johnson JM, Khoshgoftaar TM: Survey on deep learning with class imbalance. Journal of Big Data 2019, 6(1):27.

33. De Calignon A, Polydoro M, Suárez-Calvet M, William C, Adamowicz DH, Kopeikina KJ, Pitstick R, Sahara N, Ashe KH, Carlson GA: Propagation of tau pathology in a model of early Alzheimer's disease. Neuron 2012, 73(4):685-697.

34. Hyman BT, Van Hoesen GW, Damasio AR, Barnes CL: Alzheimer's disease: cell-specific pathology isolates the hippocampal formation. Science 1984, 225(4667):1168-1170. 